ARTIGO ORIGINAL ORIGINAL ARTICLE

\section{Análise de custo-efetividade do n-butil-cianoacrilato para fixação de telas em hernioplastias inguinais sob a perspectiva da saúde suplementar brasileira}

\author{
Cost-effectiveness of n-buthyl-cyanoacrylate for \\ mesh fixation in inguinal hernioplasty under the \\ perspective of Supplementary Healthcare in Brazil \\ Luciana Mensor ${ }^{1}$, Regina Contadin², Camila Pepe ${ }^{3}$, Elisanio Cardoso ${ }^{4}$ \\ DOI: $10.21115 / J B E S . v 8 . n 2 . p 132-140$
}

\section{Palavras-chave:}

custo-efetividade, hérnia inguinal, fixação de telas, n-butil-cianoacrilato

\section{Keywords:}

cost-effectiveness, inguinal hernia, mesh fixation, n-butyl-cyanoacrylate

\section{RESUMO}

Objetivo: Estimar a custo-efetividade do n-butil-cianoacrilato (NBCA) para fixação de telas em pacientes submetidos a hernioplastias inguinais por reparo aberto ou laparoscópico, sob a perspectiva da Saúde Suplementar do Brasil. Métodos: Foi elaborado modelo analítico de decisão para estimar a razão de custo efetividade incremental (RCEl) em horizonte de tempo de um ano após procedimento cirúrgico com o uso do adesivo sintético versus técnicas tradicionais de fixação (sutura e grampos). Alternativamente, o uso de NBCA foi comparado à cola de fibrina. Os desfechos clínicos avaliados foram incidência de dor aguda e crônica. A estimativa de custos médicos diretos totais para as diferentes abordagens cirúrgicas e terapia da dor foi elaborada a partir da opinião de especialistas e coletadas em bases de dados secundárias. Resultados: A RCEI obtida através do modelo evidenciou economia substancial de recursos financeiros relacionada ao uso de NBCA de aproximadamente $\mathrm{R} \$ 2.800$ por procedimento após reparo laparoscópico e $\mathrm{R} \$ 60$ reais por procedimento para reparo aberto, resultados direcionados majoritariamente pela menor tendência de dor (efetividade incremental de 21,74\% em termos de dor evitada) versus métodos de fixação tradicional. No cenário alternativo, no qual comparou-se NBCA com a cola biológica (adesivo de fibrina), atingiu-se economia financeira com nível de efetividade equivalente do ponto de vista estatístico entre os comparadores. Conclusão: Pode-se atribuir que o uso de NBCA para fixação de telas em cirurgias de reparo de hérnia inguinal constitui alternativa segura, efetiva e viável economicamente, quando comparado tanto a técnicas de fixação tradicionais como à cola biológica.

\footnotetext{
Recebido em: 24/05/2016. Aprovado para publicação em: 06/07/2016.

1. Gerente de Farmacoeconomia, Laboratórios B. Braun S/A, Rio de Janeiro, Brasil.

2. Coordenadora de Acesso ao Mercado, Laboratórios B. Braun S/A, Rio de Janeiro, Brasil.

3. Diretora de Projetos, Sense Company, São Paulo, Brasil.

4. Cirurgião Geral Adjunto do Colégio Brasileiro de Cirurgiões e Fellow da American Hernia Society, Sociedade Brasileira de Hérnias da Parede Abdominal e Federação Latino Americana de Hérnias.

Instituição onde o trabalho foi executado: Sense Company, São Paulo, Brasil.

Conflito de interesses: Luciana Lopes Mensor trabalhava nos Laboratórios B.Braun SA na época da realização do trabalho; Regina M. Contadin trabalha nos Laboratórios B.Braun S.A.; Camila Pepe trabalha na Sense Company, empresa contratada pelos Laboratórios B.Braun S.A para elaboração da Avaliação de Tecnologias em Saúde, objeto deste artigo; Elisanio S. Cardoso é consultor médico da Aesculap Academy e recebeu verbas de consultoria dos Laboratórios B.Braun S.A. para ministrar aulas e cursos sobre hernioplastia inguinal.

Autor Correspondente: Regina Maria Contadin, endereço: R. Cincinato Braga, 37, $7^{\circ}$ ANDAR São Paulo - SP, 01333-011, e-mail: regina. contadin@bbraun.com; regina.contadin@gmail.com
} 
ce of acute and chronic pain. The estimation of total direct medical costs for surgical approach and pain therapy was developed based on experts' opinions and collected from secondary databases. Results: The ICER obtained through the model indicated substantial savings of financial resources related to the use of NBCA of approximately 2,800 BRL per procedure after laparoscopic repair and $60 \mathrm{BRL}$ per procedure for open repair, results primarily due to the lower tendency of pain (incremental effectiveness of $21.74 \%$ in terms of avoided pain) versus traditional fixation methods. In the alternative scenario, which compared NBCA with biological glue (fibrin glue), it was achieved financial savings, with statistically equivalent level of effectiveness among the comparators. Conclusion: The use of NBCA for mesh fixation in inguinal hernia repair surgery is a safe, effective and feasible alternative from an economic point of view, when compared to both traditional fixation techniques as the biological glue.

\section{Introdução}

A Avaliação de Tecnologias em Saúde (ATS) define-se como um conjunto de métodos que estuda as consequências de curto, médio e longo prazos da aplicação de uma tecnologia de cuidados em saúde, fornecendo informações sobre benefícios, riscos e custos das intervenções em saúde. Para uma dada tecnologia, as propriedades a serem avaliadas incluem características técnicas, segurança, eficácia, efetividade e custo (Associação Brasileira de Farmacoeconomia e Pesquisa de Desfechos (ISPOR), 2009).

Para as hernioplastias inguinais, a escolha do tipo de reparo empregado, se aberto ou laparoscópico, é realizada pelo cirurgião, sem considerar aspectos econômicos (luamoto et al., 2015). O reparo aberto pode beneficiar os idosos e menos saudáveis, sendo dividido em dois tipos: reparo livre de tensão com a utilização de uma tela protética (normalmente polipropileno) ou reparo com tensão com a utilização de sutura, sem tela. A técnica de Lichtenstein é uma das mais comuns no reparo aberto com tela. Já o desenvolvimento das técnicas laparoscópicas revolucionou os reparos da hérnia, os quais são, atualmente, os procedimentos mais comuns realizados na cirurgia geral (Fitzgibbons \& Forse, 2015).

Em pacientes adultos, a maioria das hérnias inguinais é tratada por implantação com tela de prótese. Para impedir o deslocamento da tela e a recidiva da doença, diferentes métodos de fixação têm sido utilizados (Wang et al., 2013). Embora a reparação com sutura primária seja amplamente usada, esta abordagem de fixação apresenta inúmeras desvantagens, incluindo quantidade elevada de pontos que são necessários e o aumento da incidência de complicações, tais como dor pós-operatória, dor crônica, formação de hematoma e infecção (Paajanen, 2002; Shen et al., 2012).

Outros métodos de fixação, como grampos espirais de titânio, são também utilizados e fornecem fixações confiáveis na hernioplastia inguinal. Entretanto, apresentam complicações pós-operatórias semelhantes às verificadas com a sutura e são métodos que exigem instrumentos cirúrgicos mais onerosos (Kaul et al., 2012). Adicionalmente, adesivos biológicos, como o adesivo de fibrina, são métodos de fixação caros, que fornecem ligação fraca, possuem aplicação demorada e perfil alergênico (Fortelny et al., 2012).

A identificação de métodos que reduzam a dor pós-operatória e a infecção após a hernioplastia inguinal pode influenciar significativamente na qualidade de vida dos pacientes (Andraus et al., 2009; Pierides et al., 2013; Poobalan et al., 2001). Assim, adesivos tissulares de cianoacrilato são um método alternativo e mais recente para fixação de telas em hernioplastias inguinais, disponibilizados em uma formulação de monômero líquido e que sofrem uma reação exotérmica quando entram em contato com um meio fluido ou básico, levando à polimerização e a ligação com a pele ou órgãos (Kukleta et al., 2012; Laboratórios B.Braun S/A, n.d.).

Assim, foi desenvolvida avaliação de custo-efetividade do uso de n-butil-cianoacrilato para fixação de telas em pacientes adultos submetidos à hernioplastia inguinal por reparo aberto (cenário 1) ou laparoscópico (cenário 2), sob a perspectiva da Saúde Suplementar do Brasil.

\section{Metodologia}

A tecnologia proposta, $n$-butil-cianoacrilato (NBCA), trata-se de uma resina acrílica que se polimeriza na presença de água, especialmente com íons de hidróxido, juntando as superfícies coladas em 5-6 segundos e atingindo a fase final em 60 segundos, e se propõe a fixar telas em cirurgias de reparo aberto ou laparoscópico de hérnia inguinal, em substituição a técnicas de fixação tradicionais como as que empregam suturas ou grampos (Kukleta et al., 2012). O produto-alvo desta avaliação, Histoacryl ${ }^{\oplus}$, é um produto para saúde classe III, registrado no Brasil pelos Laboratórios B.Braun S.A.

Foi desenvolvida análise de custo efetividade (ACE) através de modelo analítico de decisão, sob a perspectiva da Saúde Suplementar do Brasil, comparando-se NBCA com técnicas tradicionais de fixação de telas em dois cenários: Cenário 1: cirurgia aberta, comparador: sutura; Cenário 2: cirurgia laparoscópica; comparador: grampos. Como cenário alternativo, o NBCA foi comparado ainda com cola biológica tanto para fixação de telas em reparo aberto de hérnia inguinal como em reparo laparoscópico. 
Como horizonte temporal, foi eleito horizonte de 1 ano, selecionado por ser o tempo necessário para determinação e manejo da dor aguda e crônica, ou um tempo suficientemente longo para refletir qualquer diferença nos custos e efetividades que estão sendo comparados. Não foi aplicada uma taxa de desconto, uma vez que o horizonte temporal escolhido foi limitado a 01 (um) ano.

Foram consideradas no modelo as definições de dor conforme Shen et ale colaboradores (Shen et al., 2012). Assim, dor aguda foi definida como a dor mensurada 24 horas após a intervenção cirúrgica. Já a dor crônica foi definida como desconforto ou dor persistente três meses após a intervenção (Shen et al., 2012). Dessa forma, as definições dos estudos clínicos que avaliaram NBCA em pacientes adultos submetidos à hernioplastia inguinal foram utilizadas na composição do modelo para sustentar o horizonte temporal da análise econômica.

Os desfechos clínicos e dados epidemiológicos utilizados no modelo econômico apresentado por este artigo foram coletados por meio de revisão sistemática da literatura científica publicada até 30/04/2015. As buscas eletrônicas seguiram a hierarquia de prioridade sugerida pela Diretriz para Elaboração de Pareceres Técnico-Científicos do Ministério da Saúde (Ministério da Saúde, Brasil, Secretaria de Ciência-Tecnologia e Insumos Estratégicos, Departamento de Ciência e Tecnologia, 2011) e as buscas foram realizadas em todas as bases obrigatórias (Registro Cochrane Central de Ensaios Controlados, The Cochrane Library, MEDLINE via Pubmed, LILACS e CRD - Centre for Reviews and Dissemination), conforme a pergunta estruturada descrita na Tabela 1. Foi ainda realizada busca complementar por estudos econômicos nas bases de dados Pubmed/MEDLINE, CRD (Centre for Reviews and Dissemination - York University/UK) e Biblioteca Virtual em Saúde - Economia da Saúde (BVS-ECOS) e em websites de agências de Avaliação de Tecnologias em Saúde, instituições correlatas e suas bases de dados.

Tabela 1. Estratégia de busca PICO

\begin{tabular}{ll}
\hline P - População & $\begin{array}{l}\text { Pacientes adultos submetidos } \\
\text { à hernioplastia inguinal. }\end{array}$ \\
\hline I - Intervenção & $\begin{array}{l}\text { Fixação de tela em hernioplastia } \\
\text { inguinal com Histoacryl } .\end{array}$ \\
\hline C - Comparação & $\begin{array}{l}\text { Fixação de tela em hernioplastia inguinal com } \\
\text { outros tipos de adesivo, grampos, sutura, etc. }\end{array}$ \\
\hline O - Desfechos & $\begin{array}{l}\text { Eficácia e segurança: sem restrição } \\
\text { de desfechos clínicos. }\end{array}$ \\
& $\begin{array}{l}\text { Avaliação econômica: utilização de } \\
\text { recursos, custo da doença, razão de } \\
\text { custo-efetividade incremental, razão } \\
\text { de custo-utilidade incremental. }\end{array}$ \\
\hline Desenho de & $\begin{array}{l}\text { Metanálises, revisões sistemáticas, ensaios } \\
\text { Clínicos, estudos observacionais e estudos de } \\
\text { Estudo }\end{array}$ \\
\hline
\end{tabular}

Na construção de estratégias de buscas, os descritores, as palavras-chave e os termos MeSH foram utilizados para cada base de dado especificamente. Não foram utilizados limites de idioma ou temporais, tampouco filtros para revisões sistemáticas ou ensaios clínicos para que as buscas fossem mais abrangentes. Apenas artigos nos idiomas português, inglês, francês, espanhol ou italiano foram incluídos.

Os estudos incluídos na revisão foram avaliados conforme a Classificação de Nível de Evidência Oxford Centre for Evidence Based Medicine (Oxford Centre for Evidence-based Medicine [CEBM], 2009). Para avaliação da qualidade metodológica das revisões sistemáticas incluídas, foi utilizada a ferramenta AMSTAR (Shea et al., 2007; 2009), ao passo que os ensaios clínicos randomizados controlados foram avaliados conforme a metodologia proposta por Jadad e colaboradores (Jadad et al., 1996).

Utilizando o software TreeAge Pro 2014 (TreeAge Software Inc., Williamstown, Estados Unidos), (TreeAge Software Inc, 2014) foi desenvolvido um modelo de decisão para estimar a razão de custo-efetividade entre NBCA e seus comparadores.

Os custos estimados aplicados ao modelo foram baseados em preços coletados de bases secundárias, considerando valores constantes das listas de Preços Fábrica (PF), (Ministério da Saúde [Brasil], 2015) disponíveis para uma taxa de Imposto sobre Circulação de Mercadorias e Serviços (ICMS) de 18\%. Custos indiretos e custos não médicos não foram considerados por não se adequarem à perspectiva selecionada. Os recursos de saúde considerados se referem a procedimentos e exames relacionados ao tratamento das reações adversas à punção venosa com cateteres periféricos, ponderados a partir das respectivas taxas de ocorrência destas reações, conforme o comparador empregado.

Os exames foram custeados conforme a Classificação Brasileira Hierarquizada de Procedimentos Médicos da Associação Médica Brasileira (CBHPM/ AMB) (Associação Médica Brasileira, 2015). Os preços de aquisição dos dispositivos médicos e produtos para a saúde foram baseados na Revista Eletrônica SIMPRO, para data base de junho de 2015 (Simpro informações e soluções em saúde, 2015). Preços de medicamentos foram coletados a partir da Lista da Câmara de Regulação de Medicamentos (CMED), também com data base de junho de 2015 (Ministério da Saúde, 2015).

Foi incluída, no modelo desenvolvido, uma coorte hipotética de pacientes adultos de ambos os sexos hospitalizados no Sistema Suplementar de Saúde Brasileiro e que necessitam se submeter a procedimento cirúrgico de hernioplastia inguinal por reparo aberto (cenário 1) ou laparoscópico (cenário 2). A estrutura do modelo pode ser visualizada na Figura 1.

Os resultados comparativos das estratégias de tratamento foram medidos pela razão de custo-efetividade incremental (RCEI), definida para duas alternativas de tratamentos específicos, como o custo adicional proporcionado pela alternativa 


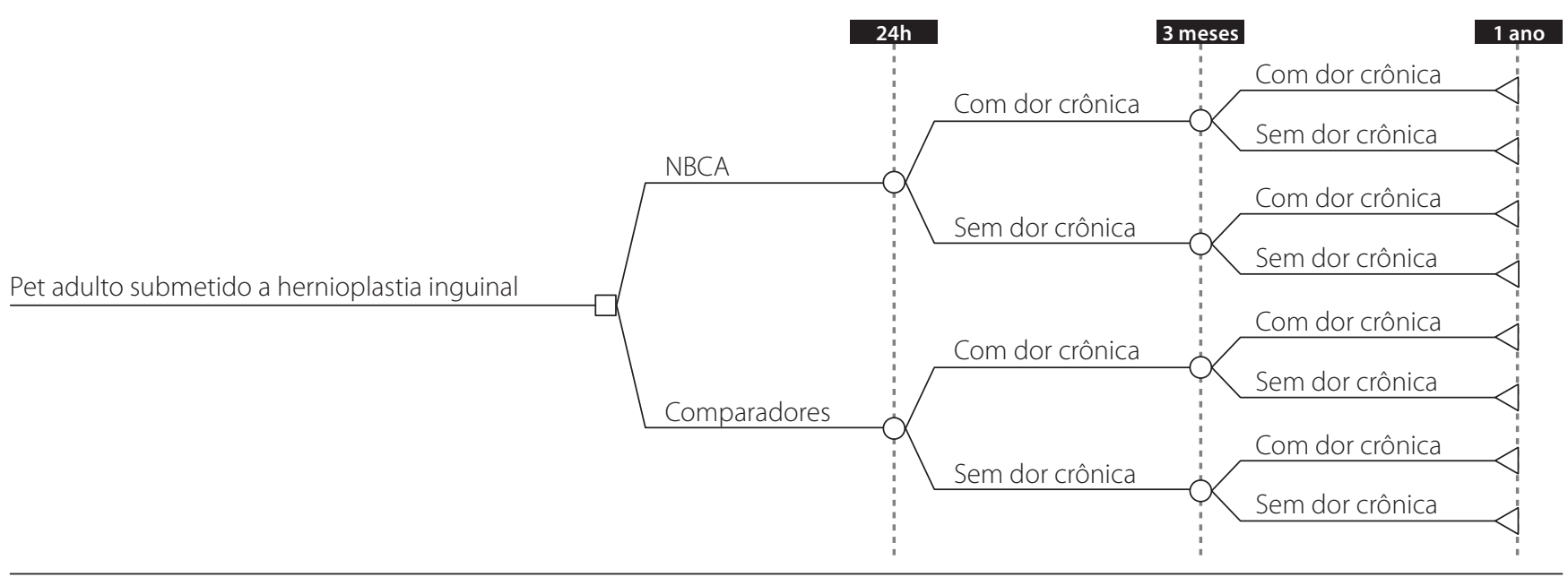

Figura 1. Modelo analítico de decisão

em análise dividido pelo ganho adicional em saúde alcançado pela mesma. Foi considerado como limiar de custo-efetividade o valor do PIB per capita no Brasil em 2014, ou R\$ 27.229 (Instituto Brasileiro de Geografia e Estatítica, 2014). Para resultados de RCEl negativa, considerar-se-á que a alternativa proposta atinge os objetivos com economia de recursos.

\section{Resultado}

Após a realização da busca nas bases de dados, 101 títulos foram localizados (incluindo duplicatas). Aplicados os critérios de elegibilidade, dois revisores selecionaram 15 estudos para leitura na íntegra. Desses, 9 estudos foram selecionados e incluídos nesta revisão.

Dentre os 9 estudos selecionados, encontram-se uma revisão sistemática (Sanders \& Waydia, 2014), cinco ensaios clínicos randomizados (Helbling \& Schlumpf, 2003; Kim-Fuchs et al., 2012; Nowobilski et al., 2004; Shen et al., 2012; Testini et al., 2010), e três estudos observacionais (Garcia-vallejo, Couto-Gonzalez, et al., 2014; Kukleta et al., 2012; Wang et al., 2013). Em relação às buscas econômicas, não foram selecionados estudos para a leitura na íntegra.

Dados de eficácia relacionados à efetividade dos comparadores foram obtidos de três estudos, sendo eles: Sanders et al., 2014 (Sanders \& Waydia, 2014) e Kaul et al., 2012 (Kaul et al., 2012) e Testini et al, 2010 (Testini et al., 2010).

O estudo de Sanders e colaboradores é uma revisão sistemática da literatura produzida a partir de estudos clínicos randomizados. Ele comparou diferentes métodos de fixação de telas na hernioplastia inguinal anterior aberta em adultos acima de 18 anos em termos de incidência de dor crônica, definida pelos autores como dor persistente por mais de 3 meses após a intervenção cirúrgica. Apesar de o autor não ter realizado uma metanálise, ele apresentou o resultado combinado de dor crônica de todos os 12 estudos clínicos inclu- ídos: $14,7 \%$ para suturas, 7,6\% para NBCA e 3,7\% para adesivo de fibrina (Sanders \& Waydia, 2014).

No entanto, dos doze estudos incluídos na revisão sistemática de Sanders e colaboradores, nem todos os estudos apresentavam qualidade metodológica considerada aceitável pelos revisores do presente trabalho. Assim, com o objetivo de utilizar somente as evidências de melhor qualidade no modelo desenvolvido, foram excluídos os estudos de qualidade MUITO BAIXA (6 artigos), permanecendo apenas aqueles de qualidade MODERADA (5 artigos) e BAIXA (1 artigo). Os dados desse ajuste estão apresentados na Tabela 2.

Como a revisão de Sanders não incluiu nenhum estudo que avaliasse o grampo, foi necessária a implementação de uma comparação indireta. A bibliografia apresentada nos estudos incluídos na revisão sistemática elaborada para este projeto foi consultada com o objetivo de identificar estudos específicos de grampo. Foi identificado o estudo de Kaul e colaboradores, o qual foi utilizado para a implementação da comparação indireta (Kaul et al., 2012). Os autores avaliaram a persistência de dor crônica por mais de 3 meses após a intervenção, comparando a fixação da tela usando adesivo de fibrina com a fixação com uso de grampo no procedimento de hernioplastia inguinal laparoscópica (Kaul et al., 2012). O odds ratio (OR) da comparação de adesivo de fibrina versus grampo reportado foi de 3,25 (Kaul et al., 2012). Tendo este OR e a incidência de dor crônica ajustada do estudo de Sanders para pacientes em uso de adesivo de fibrina $(8,1 \%)$, foi

Tabela 2. Incidência de dor crônica ajustada para os comparadores NBCA, sutura e adesivo de fibrina

\begin{tabular}{lc}
\hline Comparador & \% de dor crônica \\
\hline NBCA & $10,1 \%$ \\
\hline Sutura & $20,2 \%$ \\
\hline Adesivo de fibrina & $8,1 \%$ \\
\hline
\end{tabular}


calculada a incidência de dor crônica em pacientes em uso do grampo, sendo esta a multiplicação destes valores $(8,1 \%$ e 3,25), resultando numa incidência de $26,3 \%$.

Os padrões de custo de tratamento para cada linha e subgrupo de tratamento foram desenvolvidos através da opinião de especialistas e custeados de acordo com a sua incidência de utilização dos recursos, quantidade e preço unitário. Os

Tabela 3. Custos

\begin{tabular}{lc}
\hline Item de custeio & Custo Total \\
\hline Dor aguda & $R \$ 1.495,44$ \\
\hline Dor crônica & $R \$ 8.296,83$ \\
\hline Reparo cirúrgico da hérnia - cirurgia aberta & $R \$ 6.172,57$ \\
\hline $\begin{array}{l}\text { Reparo cirúrgico da hérnia } \\
\text { - videolaparoscopia }\end{array}$ & $R \$ 7.844,00$ \\
\hline NBCA (para reparo aberta): 01 unidade & $R \$ 1.000,00$ \\
\hline $\begin{array}{l}\text { NBCA (para reparo laparoscópico, } \\
\text { incluindo cateter de peridural como } \\
\text { aplicador): 01 unidade de cada }\end{array}$ & $R \$ 1.160,26$ \\
\hline Sutura* & $R \$ 91,09$ \\
\hline Adesivo fibrina: 01 unidade† & $R \$ 2.377,03$ \\
\hline Grampo & $R \$ 2.481,70$ \\
\hline
\end{tabular}

* Foi realizada a média de preços das principais suturas comercializadas no país e considerado o uso de duas unidades de sutura por procedimento.

†Já comercializado com aplicador. valores unitários dos itens considerados para cada estratégia de tratamento são visualizados na Tabela 3.

Após a aplicação de todos os vetores supracitados de custo e eficácia clínica dentro do modelo econômico desenvolvido, chegamos aos resultados das razões de custo efetividade incrementais (RCEI) para cada cenário considerado (Tabela 4 e Tabela 5). Alternativamente, ambos os cenários foram analisados conforme o tipo cirúrgico, variando-se a técnica de fixação de telas para o uso de cola biológica (adesivo de fibrina). Os resultados desta análise alternativa podem ser observados na Tabela 6 e Tabela 7.

\section{Cenários base 1 e 2}

Os resultados da análise do cenário 1 demonstraram que o custo do procedimento de cirurgia aberta de hernioplastia inguinal com o uso de NBCA foi ligeiramente menos oneroso comparado com o uso de sutura (- $R \$ 59,00$ ), sendo que os pacientes apresentaram menor índice de dor pós-operatória com o uso de NBCA em ambos os desfechos da análise. Assim, observamos valores de RCEI negativos (ou cost-saving) de $-\mathrm{R} \$ 579,41$ e $-\mathrm{R} \$ 362,13$ por desfecho evitado, paciente livre de dor crônica e paciente livre de dor, respectivamente. Ou seja, obtém-se economia de aproximadamente R\$ 580 para cada caso de dor crônica evitado ou de cerca de $R \$ 362$ para cada caso de dor evitado (aguda e crônica).

Tabela 4. Razão de custo efetividade do caso base da análise de cenário1: NBCA versus sutura

\begin{tabular}{llll}
\hline Comparador & NBCA & Sutura & $\begin{array}{l}\text { Incremental } \\
\text { (NBCA vs. Sutura) }\end{array}$ \\
\hline Custo total do procedimento* & $R \$ 8.063,39$ & $\mathrm{R} \$ 8.122,49$ & $-\mathrm{R} \$ 59,10$ \\
\hline \% livre de dor crônica & $89,93 \%$ & $79,73 \%$ & $10,20 \%$ \\
\hline \% livre de dor (aguda e crônica) & $86,60 \%$ & $70,28 \%$ & $16,32 \%$ \\
\hline Razão de Custo-Efetividade Incremental (NBCA vs. sutura) & & & \\
\hline RCEI PACIENTE LIVRE DE DOR CRÔNICA & & Cost-saving \\
& & & (-R\$ 579,41) \\
\hline RCEI PACIENTE LIVRE DE DOR & & Cost-saving \\
\end{tabular}

* Neste custo estão inclusos o custo da cirurgia e o custo do material empregado para fixação das telas.

Tabela 5. Razão de custo efetividade para o caso base do cenário 2: NBCA versus grampo

\begin{tabular}{llll}
\hline Comparador & NBCA & Grampo & $\begin{array}{l}\text { Incremental } \\
\text { (NBCA vs. grampo) }\end{array}$ \\
\hline Custo total do procedimento* & $R \$ 9.895,08$ & $\mathrm{R} \$ 12.694,85$ & $-\mathrm{R} \$ 2.799,77$ \\
\hline \% livre de dor crônica & $89,93 \%$ & $73,58 \%$ & $16,35 \%$ \\
\hline \% livre de dor (aguda e crônica) & $86,60 \%$ & $64,86 \%$ & $21,74 \%$ \\
\hline Razão de Custo-Efetividade Incremental (NBCA vs. grampo) & & & \\
\hline RCEI PACIENTE LIVRE DE DOR CRÔNICA & & Cost-saving \\
& & & (-R\$ 17.123,98) \\
\hline RCEI PACIENTE LIVRE DE DOR & & Cost-saving \\
& & & $(-R \$ 12.878,43)$ \\
\hline
\end{tabular}

* Neste custo está incluso o custo da cirurgia mais o custo do fixador. 
Tabela 6. Razão de custo efetividade do caso alternativo do cenário 1: NBCA versus adesivo de fibrina em reparo aberto

\begin{tabular}{llll}
\hline Comparador & NBCA & Fibrina & $\begin{array}{l}\text { Incremental } \\
\text { (NBCA vs. fibrina) }\end{array}$ \\
\hline Custo total do procedimento* & $R \$ 8.063,39$ & $R \$ 9.276,49$ & $-R \$ 1.213,10$ \\
\hline$\%$ livre de dor crônica & $89,93 \%$ & $91,93 \%$ & $-2,00 \%$ \\
\hline$\%$ livre de dor (aguda e crônica) & $86,60 \%$ & $88,39 \%$ & $-1,79 \%$ \\
\hline Razão de Custo-Efetividade Incremental (NBCA vs. fibrina) & & & Menos oneroso com efetividade similar \\
\hline RCEI PACIENTE LIVRE DE DOR CRÔNICA & & & Menos oneroso com efetividade similar \\
\hline RCEI PACIENTE LIVRE DE DOR & & & \\
\hline
\end{tabular}

* Neste custo está incluso o custo da cirurgia mais o custo do fixador.

Tabela 7. Razão de custo efetividade do caso alternativo do cenário 2: NBCA versus adesivo de fibrina em reparo laparoscópico

\begin{tabular}{llll}
\hline Comparador & NBCA & $\begin{array}{l}\text { Adesivo de } \\
\text { fibrina }\end{array}$ & $\begin{array}{l}\text { Incremental } \\
\text { (NBCA vs. Adesivo de fibrina) }\end{array}$ \\
\hline Custo total do procedimento* & $R \$ 8.223,65$ & $\mathrm{R} \$ 9.276,49$ & $-\mathrm{R} \$ 1.052,84$ \\
\hline \% livre de dor crônica & $89,93 \%$ & $91,93 \%$ & $-2,00 \%$ \\
\hline \% livre de dor (aguda e crônica) & $86,60 \%$ & $88,39 \%$ & $-1,79 \%$ \\
\hline Razão de Custo-Efetividade Incremental (NBCA vs. fibrina) & & & \\
\hline RCEI PACIENTE LIVRE DE DOR CRÔNICA & & Menos oneroso com efetividade similar \\
\hline RCEI PACIENTE LIVRE DE DOR & & Menos oneroso com efetividade similar \\
\hline
\end{tabular}

* Neste custo está incluso o custo da cirurgia mais o custo do fixador.

Os resultados da análise do cenário 2 demonstraram que o custo do procedimento de cirurgia laparoscópica de hernioplastia inguinal com o uso de NBCA foi bem menos oneroso comparado com o uso de grampo e, adicionalmente, em relação à efetividade, os pacientes apresentaram menor índice de dor pós-operatória com o uso de NBCA em ambos os desfechos da análise. Ou seja, o resultado da análise de custo-efetividade gerou uma RCEI negativa (cost-saving) de $-\mathrm{R} \$ 17.123,98$ e $-\mathrm{R} \$ 12.878,43$ por desfecho evitado, respectivamente pacientes livre de dor crônica e pacientes livre de dor. A interpretação destes números nos mostra que uma economia de aproximadamente $\mathrm{R} \$ 17,1$ mil é esperada para cada caso de dor crônica evitado. Da mesma forma, espera-se uma economia média de aproximadamente $\mathrm{R} \$ 12,8$ mil para cada caso de dor evitado, independentemente do tipo de a dor ser aguda ou crônica.

\section{Cenário alternativo}

Os resultados da análise demonstraram que o custo do procedimento de cirurgia aberta de hernioplastia inguinal com o uso de NBCA foi menos oneroso quando comparado com o uso de adesivo de fibrina e, em relação à efetividade, os pacientes apresentaram um maior, porém pouco representativo, índice de dor pós-operatória com o uso de NBCA. Se fizermos o cálculo da Razão de Custo-Efetividade Incremental (RCEI) inverso, comparando Fibrina ao NBCA ao invés de NBCA com fibrina, teremos o valor de $\mathrm{R} \$ 60.655,00$ por paciente livre de dor crônica e $\mathrm{R} \$ 67.770,95$ por paciente livre de dor. Ou seja, um custo incremental de R\$ 1.213 para se obter o benefício de evitar aproximadamente $2 \%$ dos casos de dor, gera uma RCEI muita alta, apontando que o custo adicional não justifica o benefício alcançado pelo paciente.

Mais uma vez, os resultados da análise de cenário alternativo demonstraram que o custo do procedimento de cirurgia laparoscópica de hernioplastia inguinal com o uso de NBCA foi menos oneroso comparado com o uso de adesivo de fibrina e, em relação à efetividade, os pacientes apresentaram um maior, porém não significante, índice de dor pós-operatória com o uso de NBCA. Se fizermos o cálculo da Razão de Custo-Efetividade Incremental (RCEI) comparando Fibrina ao NBCA ao invés de NBCA com fibrina, teremos o valor de $\mathrm{R} \$ 52.642,00$ por paciente livre de dor crônica e R\$ 58.817,88 por paciente livre de dor. Ou seja, um custo incremental de R\$ 1.052,84 para se obter o benefício de evitar aproximadamente $2 \%$ dos casos de dor, gera uma RCEl muita alta, apontando que o custo adicional não justifica o benefício alcançado pelo paciente.

Um importante elemento em um estudo econômico para a tomada de decisão é a quantificação da incerteza envolvida nos seus resultados e a identificação das variáveis que mais afetam esta incerteza. Portanto, foram realizadas análises de sensibilidade para melhor compor o resultado da análise. Apesar da variação de 10\% dos parâmetros avaliados na análise, os resultados finais do modelo não sofreram alteração. 


\section{Discussão}

As análises econômicas em saúde são ferramentas eficientes para suporte à alocação de recursos para gestores de sistema de saúde, pois oferecem projeções no longo prazo. A contribuição fundamental dos modelos econômicos específicos para cada país é utilizar dados de custos e padrões de tratamento da prática médica local, auxiliando assim aos tomadores de decisão na tarefa de estimar o impacto financeiro de uma inovação em saúde, avaliando de forma mais abrangente a viabilidade da sua incorporação.

Foi realizada busca por estudos econômicos publicados para NBCA nas bases de dado Medline, Embase, The Cochrane Library, LILACS, CDR (Centre for Reviews and Dissemination e BVS-ECOS (Biblioteca Virtual em Saúde - Econômica da Saúde). Não foram selecionados estudos para a leitura íntegra, ou seja, não temos resultados já publicados na literatura para que possamos comparar com os achados do trabalho objeto deste estudo. Da mesma forma, como não temos estudos publicados sobre o tema, não foi possível replicar uma estrutura já desenhada e validada de modelo econômico por outros autores. Assim, foi considerado um desenho de modelo que reflete a realidade brasileira, tanto no que diz respeito aos comparadores selecionados como aos custos unitários que serviram de input para a avaliação econômica. Para isso, alguns profissionais da área da saúde, que trabalham atualmente com a patologia em questão, foram consultados para validar a estrutura considerada.

Com relação aos dados clínicos que embasam o modelo desenvolvido, para melhor avaliar a robustez dos resultados encontrados por Sanders (Sanders \& Waydia, 2014), optamos por aplicar um teste estatístico nos dados fonte, especialmente para verificar se as incidências observadas de dor crônica seriam estatisticamente significantes. Foi empregado para tal o teste T-student, que compara duas médias em questão, usando o portal mathportal. Os resultados para a comparação NBCA x sutura apontaram um valor t de 1,6952 e de 0,2624 para a comparação NBCA x adesivo de fibrina, com valores críticos de 2,306 e 3,182, respectivamente. O valor crítico referenciado trata-se de um valor tabelado considerando um nível de confiança de 95\% e o tamanho de cada amostra. Se t-valor < valor crítico, então o resultado é não significante.

Dessa forma, surpreendentemente, nenhuma das duas comparações mostrou-se significante. Tal resultado já era esperado na comparação NBCA $x$ adesivo de fibrina, já que os dados percentuais de incidência de dor crônica são muito próximos e aparentemente apresentam diferença irrelevante do ponto de vista clínico (8,10\% para adesivo de fibrina e 10,07\% para NBCA). Porém, na comparação NBCA e sutura, com dados fonte de 20,19\% de incidência de dor crônica para o grupo sutura e 10,07\% para o NBCA, o resultado de não significância mostrou-se intrigante. Uma das possíveis justifica- tivas desse resultado poderia residir no número limitado de pacientes ( $N=660)$, associado ao pequeno número de estudos de base (apenas 3 em comparação direta foram elencados na metanálise de Sanders), além da ampla variabilidade de resultados e metodologias empregadas nos mesmos.

Pode-se perceber pela revisão de literatura, no entanto, que há uma grande tendência de redução de dor aguda e crônica associada ao uso de adesivos (sintéticos ou biológicos) ao invés de técnicas tradicionais como suturas e grampos, amplamente relatadas em estudos publicados na literatura correlata (Campanelli et al., 2012; Helbling \& Schlumpf, 2003; Kapischke et al., 2010; Lionetti et al., 2012; Nowobilski et al., 2004). A justificativa técnica para tal é plausível, já que o dano a nervos periféricos com o uso dos adesivos é potencialmente menor do que empregando-se grampos ou sutura, em especial quando se trata da região abdominal chamada de "triângulo da dor" (Fränneby et al., 2006; Liu et al., 2014). Se realizarmos inferência indireta, por exemplo, baseada nos resultados de recente metanálise publicada por Liu e colaboradores em 2014 (Liu et al., 2014), podemos chegar a resultados díspares à análise estatística realizada pelo nosso grupo. Liu e colaboradores, nesta metanálise, mostram dados de risco relativo significativos (RR 0.42, 95\% Cl 0.22-0.79, I 2 11\%; $p<0.01$ ) na incidência de dor crônica associada ao uso de sutura para fixação de telas em hernioplastias inguinais, quando comparado com adesivo de fibrina ( $N=1.623$ pacientes).

Outra questão seria a comparação indireta entre NBCA e o uso de grampos em cirurgia laparoscópica. Neste caso, o teste estatístico não foi aplicado, já que o estudo Kaul e colaboradores reportou o OR utilizado para o cálculo da comparação indireta já com intervalo de confiança (IC) 95\% (OR: 3,25; IC95\%: 1,62-6,49) (Kaul et al., 2012). Este resultado mostra que a diferença entre o uso de grampo e adesivo de fibrina é estatisticamente significante, pois os limites do IC 95\% são maiores do que 1. Como é indubitável que não existe diferença entre o uso de NBCA e adesivo de fibrina, pode-se concluir que existe diferença estatisticamente significante entre NBCA e grampo.

Com relação à dor aguda, o estudo de Testini e colaboradores avaliou a incidência de dor 24 horas após a cirurgia de reparo da hérnia inguinal com uso de suturas, adesivo de fibrina ou NBCA para fixação da tela. No total foram incluídos 156 pacientes, sendo 12 mulheres e 144 homens, com idade de 58 anos (intervalo, 17-85 anos) (Testini et al., 2010). O p-valor reportado no estudo mostra que a diferença entre NBCA e sutura (incidência de dor de 3,7\% e 11,9\%, respectivamente, $\mathrm{p}=0,004$ ) é estatisticamente significante (ou seja, $p$-valor < $0,05)$, ao passo que na comparação entre NBCA e adesivo de fibrina (incidência de dor de 3,7\% e 3,8\%, $p=0,79$ ) a diferença não é significante (ou seja, $\mathrm{p}$-valor $>0,05$ ).

Mais uma vez aqui fica evidente que a efetividade dos comparadores alternativos, NBCA e adesivo de fibrina, mos- 
tram-se equivalentes no que se refere à incidência de dor. Quando comparados a técnicas tradicionais, existe significativa redução de dor associada ao uso de adesivos quando comparados a suturas, por exemplo.

Futuros estudos clínicos com maior número de pacientes e metodologias mais robustas em comparação direta entre tecnologias poderão atestar de forma inequívoca os benefícios dos adesivos como alternativas seguras, eficazes e viáveis ao uso de grampos e suturas durante fixação de telas em hernioplastias inguinais.

Os resultados da análise econômica realizada pelo nosso grupo foram favoráveis ao procedimento cirúrgico com NBCA. O modelo desenvolvido avaliou qual técnica de fixação de tela no procedimento de hernioplastia inguinal seria mais custo-efetiva, para o que dois cenários foram criados. Tais cenários foram diferenciados pelo tipo de cirurgia empregada no reparo da hérnia: reparo aberto ou cenário 1; reparo laparoscópico ou cenário 2.

Apesar de apresentarem custos unitários diferentes, as razões de custo efetividade incremental foram semelhantes em ambos os cenários analisados. Para o cenário 1, a comparação da fixação de tela com NBCA versus a sutura apontou que o uso do NBCA é cost-saving, ou seja, NBCA é menos oneroso e traz mais benefício clínico para o paciente. Os resultados de RCEl evidenciam uma economia média de aproximadamente $\mathrm{R} \$ 362$ para cada caso de dor evitado, independentemente do tipo da dor, aguda ou crônica. Da mesma forma, no cenário 2, observamos resultado favorável em ambos os desfechos, ou seja, NBCA é menos oneroso e traz mais benefício clínico para o paciente quando comparado a grampos para fixação das telas. Ou seja, os resultados de RCEl evidenciam que é esperada uma economia média de aproximadamente $\mathrm{R} \$ 12,8$ mil para cada caso de dor evitado, independentemente do tipo da dor, aguda ou crônica.

$\mathrm{Na}$ análise de cenários alternativos, quando o NBCA é comparado à cola de fibrina, seja por via de reparo aberto ou laparoscópico, observamos resultados de um custo relevantemente menor para a técnica com NBCA, porém com melhor ganho de efetividade para a fibrina, apesar de não significante. Ao analisar os dados de forma reversa, ou seja, avaliarmos uma eventual incorporação da cola de fibrina comparada ao NBCA, temos uma RCEI de R\$ 67,8 mil por paciente livre de dor no seu uso em reparo aberto e $R \$ 58,8$ mil para seu uso em reparo laparoscópico. Ou seja, para obter-se o benefício de evitar aproximadamente $2 \%$ dos casos de dor, gera-se uma RCEI muita alta, entre 2 e 3 vezes o PIB per capita do país ${ }^{a}$, indicando que o custo adicional da cola de fibrina

a Foi considerado como limiar de custo-efetividade nesta análise o PIB per capita no Brasil, no valor de R\$ 27 mil (2014). Considera-se como extremamente custo-efetiva a intervenção que produza RCEI < 1x PIB per capita. Se RCEI > 3x PIB per capita, a intervenção é considerada não custo-efetiva. em oposição ao NBCA poderia não justificar o benefício proposto pela mesma.

\section{Conclusão}

A partir da análise econômica realizada, credita-se ao uso do NBCA na fixação de telas em hernioplastias inguinais de pacientes adultos, seja por via aberta ou laparoscópica, significativa contribuição na redução da dor, aguda e crônica, motivo pelo qual sua incorporação estaria em linha com as recentes políticas que buscam aprimorar a saúde e a qualidade de vida dos pacientes do país.

\section{Limitações}

Os dados de eficácia clínica aplicados no modelo econômico discutido por este artigo são oriundos de revisões sistemáticas e metanálises recentes e de estudos randomizados que comparam diretamente as alternativas em destaque, publicados até a data de corte da etapa de revisão de literatura. Porém, não há como garantir que todos os estudos que se encaixaram nos critérios de inclusão tenham sido selecionados em função, sobretudo, de diferentes metodologias de indexação empregadas pelas bases de dados pesquisadas, do efeito gaveta (file drawer effect: estudos conduzidos, mas nunca publicados) e da literatura cinzenta (grey literature: informações produzidas e distribuídas em diversos níveis, em formato impresso ou eletrônico, não controladas, por publicações comerciais).

Com relação à validade externa do modelo, cabe ressaltar que todos os valores empregados para cálculo de materiais de consumo a partir dos Preços Fábrica publicados para o produto Histoacryl, registrado no Brasil pelos Laboratórios B.Braun S.A., o que pode determinar resultados diferentes caso sejam considerados outros produtos e/ou sistemas concorrentes.

Por fim, não foi considerada a inclusão de dados referentes a custos indiretos ou intangíveis, tampouco despesas com recursos humanos, em função destes vetores de custos não serem apropriados à perspectiva da avaliação realizada.

\section{Referências bibliográficas}

1. Andraus W, Paoletti B, Pinheiro R, Bitencourt F, Farias C, D'Albuquerque L. Avaliação da qualidade de vida em pacientes cirróticos com hérnia da parede abdominal. ABCD Arq Bras Cir Dig. 2009;22(4):222-5.

2. Associação Brasileira de Farmacoeconomia e Pesquisa de Desfechos (ISPOR). Custo em saúde, qualidade e desfechos: O livro de termos da ISPOR. São Paulo: Ispor Brasil; 2009.

3. Associação Médica Brasileira (AMB). Classificação Brasileira Hierarquizada de Procedimentos Médicos (CBHPM).São Paulo: AMB; 2015.

4. Campanelli G, Pascual MH, Hoeferlin A, Rosenberg J, Champault G, Kingsnorth A, Miserez M. Randomized, controlled, blinded trial of Tisseel/ Tissucol for mesh fixation in patients undergoing Lichtenstein technique for primary inguinal hernia repair: results of the TIMELI trial. Annals of Surgery. 2012;255(4):650-7. 
5. Fitzgibbons RJ, Forse RA. Groin hernias in adults. N Engl J Med. 2015;372(8):756-763.

6. Fortelny RH, Petter-Puchner AH, Glaser KS, Redl H. Use of fibrin sealant (Tisseel/Tissucol) in hernia repair: a systematic review. Surg Endosc. 2012;26(7):1803-1812.

7. Fränneby U, Sandblom G, Nordin P, Nyrén O, Gunnarsson U. Risk factors for long-term pain after hernia surgery. Annals of Surgery. 2006;244(2):212-219.

8. Garcia-Vallejo L, Couto-Gonzalez I, Concheiro-Coello P, Brea-Garcia B, Taboada-Suarez A. Cyanoacrylate surgical glue for mesh fixation in laparoscopic total extraperitoneal hernia repair. Surg Laparosc Endosc Percutam Tech. 2014;24(3): 240-243.

9. Helbling C, Schlumpf R. Sutureless Lichtenstein: first results of a prospective randomised clinical trial. Hernia. 2003;7(2):80-84.

10. Instituto Brasileiro de Geografia e Estatítica (IBGE). PIB per capita [site na internet]. 2014 [atualizado em 2014; citado 2016 mar 14]. Disponível em: http://cod.ibge.gov.br/4501p

11. Iuamoto L, Kato J, Meyer A, Blanc P. Hernioplastia laparoscópica totalmente extraperitoneal (TEP) utilizando dois trocárteres: reparos anatômicos e técnica cirúrgica. ABCD Arq Bras Cir Dig. 2015; 28(2):121-123.

12. Jadad A, Moore R, Carroll D, Jenkinson C, Reynolds D, Gavaghan D, McQuay H. Assessing the quality of reports of randomized clinical trials: is blinding necessary? Controlled Clinical Trials. 1996;17(1):1-12.

13. Kapischke M, Schulze H, Caliebe A. Self-fixating mesh for the Lichtenstein procedure--a prestudy. Langenbeck's Archives of Surgery. 2010;395(4):317-22.

14. Kaul A, Hutfless S, Le H, Hamed SA, Tymitz K, Nguyen H, Marohn MR. Staple versus fibrin glue fixation in laparoscopic total extraperitoneal repair of inguinal hernia: a systematic review and meta-analysis. Surg Endosc. 2012;26(5):1269-1278.

15. Kim-Fuchs C, Angst E, Vorburger S, Helbling C, Candinas D, Schlumpf R. Prospective randomized trial comparing sutured with sutureless mesh fixation for Lichtenstein hernia repair: long-term results. Hernia. 2012;16(1): 21-27.

16. Kukleta JF, Freytag C, Weber M. Efficiency and safety of mesh fixation in laparoscopic inguinal hernia repair using n-butyl-cyanoacrylate: long-term biocompatibility in over 1, 300 mesh fixations. Hernia. 2012;16(2):153-162.

17. Laboratórios B.Braun S/A. (n.d.). Histoacryl ${ }^{\oplus}$ - Adesivo Cirúrgico [Bula].

18. Lionetti R, Neola B, Dilillo S, Bruzzese D, Ferulano GP. Sutureless hernioplasty with light-weight mesh and fibrin glue versus Lichtenstein procedure: a comparison of outcomes focusing on chronic postoperative pain. Hernia. 2012;16(2),127-31.

19. Liu H, Zheng X, Gu Y, Guo S. A meta-analysis examining the use of fibrin glue mesh fixation versus suture mesh fixation in open inguinal hernia repair. Digestive Surgery. 2014;31(6):444-451.

20. Ministério da Saúde (Brasil). Secretaria de Ciência, Tecnologia e Insumos Estratégicos. Departamento de Ciência e Tecnologia. Diretrizes metodológicas: elaboração de pareceres técnico-científicos. (3 ed.). Brasilia: Ministério da Saúde, 2011.80 p.
21. Ministério da Saúde. (Brasil).Câmara de Regulação do Mercado de Medicamentos (CMED). [site na internet]. 2015. [atualizado em 2015; citado 2016 mar 14]. Disponível em: http://portal.anvisa.gov.br/wps/ content/Anvisa+Portal/Anvisa/Post-+Comercializacao+-+Pos+-+Uso/ Regulacao+de+Marcado

22. Nowobilski W, Dobosz M, Wojciechowicz T, Mionskowska L. Lichtenstein inguinal hernioplasty using butyl-2-cyanoacrylate versus sutures: preliminary experience of a prospective randomized trial. European Surgical Research. 2004;36(6):367-370.

23. Oxford Centre for Evidence-based Medicine (CEBM). (2009). Oxford Centre for Evidence-based Medicine - Levels of Evidence. [site na internet]. 2009. [atualizado em 2009; citado 2016 mar 14]. Disponível em: http://www.cebm.net/oxford-centre-evidence-based-medicine-levelsevidence-march-2009/

24. Paajanen, H. Do absorbable mesh sutures cause less chronic pain than nonabsorbable sutures after Lichtenstein inguinal herniorraphy? Hernia.2002;6(1):26-28.

25. Pierides G, Mattila K, Vironen J. Quality of life change in elderly patients undergoing open inguinal hernia repair. Hernia. 2013;17(6):729-736.

26. Poobalan AS, Bruce J, King PM, Chambers WA, Krukowski ZH, Smith WC. Chronic pain and quality of life following open inguinal hernia repair. $\mathrm{Br}$ J Surg. 2001;88(8):1122-6.

27. Sanders DL, Waydia S. A systematic review of randomised control trials assessing mesh fixation in open inguinal hernia repair. Hernia. 2014;18(2):165-176.

28. Shea BJ, Grimshaw JM, Wells GA, Boers M, Andersson N, Hamel C, Bouter LM. Development of AMSTAR: a measurement tool to assess the methodological quality of systematic reviews. BMC Medical Research Methodology. 2007; 7:10.

29. Shea BJ, Hamel C, Wells GA, Bouter LM, Kristjansson E, Grimshaw J, Boers M. AMSTAR is a reliable and valid measurement tool to assess the methodological quality of systematic reviews. Journal of Clinical Epidemiology. 2009;62(10):1013-20.

30. Shen YM, Sun WB, Chen J, Liu SJ, Wang MG. NBCA medical adhesive (n-butyl-2-cyanoacrylate) versus suture for patch fixation in Lichtenstein inguinal herniorrhaphy: A randomized controlled trial. Surgery. 2012;151(4):550-555.

31. Simpro informações e soluções em saúde. Revista Simpro Hospitalar. 2015.

32. Testini M, Lissidini G, Poli E, Gurrado A, Lardo D, Piccinni G. A singlesurgeon randomized trial comparing sutures, $\mathrm{N}$-butyl-2-cyanoacrylate and human fibrin glue for mesh fixation during primary inguinal hernia repair. Canadian Journal of Surgery. 2010;53(3), 155-160.

33. TreeAge Software Inc. TreeAge Pro [site na internet]. 2014. [atualizado em 2014; citado 2016 mar 14]. Disponível em: https://www.treeage.com.

34. Wang MG, Tian ML, Zhao XF, Nie YS, Chen J, ShenYM. Effectiveness and safety of n-butyl-2-cyanoacrylate medical adhesive for noninvasive patch fixation in laparoscopic inguinal hernia repair. Surg Endosc. 2013;27(10):3792-3798. 